\title{
Detección de Aconophora compressa Walker (Hemiptera: Membracidae) en poblaciones de Pinus greggii. Nuevo reporte en la Sierra Fría, Aguascalientes, México
}

\author{
Detection of Aconophora compressa Walker (Hemiptera: Membracidae) in \\ populations of Pinus greggii. New report from the Sierra Fria, Aguascalientes, Mexico

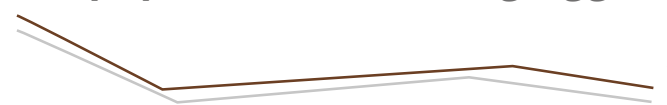 \\ Vicente Díaz-Núñez*凶, Joaquín Sosa-Ramírez**, \\ Andrés Herrera-Rodríguez****, María Guadalupe Luna-Arvizu***** \\ Díaz-Núñez, V., Sosa-Ramírez, J., Herrera-Rodríguez, A., \& Luna-Arvizu, M. G. (2018). \\ Detección de Aconophora compressa Walker (Hemiptera: Membracidae) en \\ poblaciones de Pinus greggii. Nuevo reporte en la Sierra Fría, Aguascalientes, México. \\ Investigación y Ciencia de la Universidad Autónoma de Aguascalientes, 26(73), 13-19.
}

\section{RESUMEN}

La invasión de especies biológicas es uno de los disturbios con mayores efectos negativos en los ecosistemas. En el Área Natural Protegida Sierra Fría, durante el 2013 se observó un insecto chupador afectando una población de Pinus greggii var. greggii. Para localizar más sitios infestados se realizaron recorridos por cuatro localidades donde se usó esta especie para reforestaciones. El insecto fue identificado usando claves específicas para el orden Membracidae y corroborado con especialistas en taxonomía. Para analizar la posible dispersión del insecto se realizó un monitoreo durante dos años en sitios con reforestaciones donde se usó $P$. greggii var. greggii. El insecto se identificó como Aconophora compressa Walker, una especie usada para el control biológico de plantas arvenses en

Palabras clave: especies invasoras; control biológico; reforestación; Pinus greggii; plaga forestal.

Keywords: invasive species; biological control; reforestation; Pinus greggii; forest pest.

Recibido: 21 de julio de 2017, aceptado: 27 de octubre de 2017

* Invitación de la Universidad Autónoma de Aguascalientes. Av. Universidad 940, Ciudad Universitaria, C. P. 20131, Aguascalientes, Aguascalientes, México. Correo electrónico: vicente-diaz@hotmail. com

** Centro de Ciencias Agropecuarias, Universidad Autónoma de Aguascalientes. Km. 3.5 Carr. Jesús María-Ejido San Lorenzo, C. P. 20920, Jesús María, Aguascalientes, México. Correo electrónico: jsosar@correo.vaa.mx

*** Comisión Nacional Forestal. Calle María Concepción Maldonado No. 301, C. P. 20040, Fracc. La Concordia, Aguascalientes, México. Correo electrónico: andresherrera_54@yahoo.com.mx

**** Comisión Nacional de Áreas Naturales Protegidas Aguascalientes. Av. Francisco I. Madero 718, C. P. 20259, Aguascalientes, México. Correo electrónico: mariag.luna@conanp.gob.mx

$凶$ Autor para correspondencia cultivos tropicales. Durante los últimos dos años no se han observado nuevas infestaciones. Los resultados indican que antes de realizar cualquier reforestación debe realizarse una revisión exhaustiva del estado fitosanitario de las plantas que se usarán.

\section{ABSTRACT}

The introduction of biological species is a disturbance with some of the most negative effects on ecosystems. In the Sierra Fria-Natural Protected Area-, in the year 2013 , the damage of a sucking insect was observed affecting a population of Pinus greggii var. greggii. To identify more infested sites, four places were surveyed where this species was used in afforestation. The insect was identified using specific taxonomic clues to the order Membracidae and corroborated with specialists. To study the possible dispersion of the insect, for two years reforested sites where $P$. greggii var. greggii was used were monitored. The insect was identified as Aconophora compressa Walker, comonly used for the biological control of arable plants in tropical crops. During the last two years new infestations have not been observed. The results indicate that before carrying out any reforestation, a comprehensive review of the phytosanitary status of the vegetation to be used should be undertaken.

\section{INTRODUCCIÓN}

La invasión de especies biológicas es una de las principales amenazas a la biodiversidad, a la conservación de los ecosistemas y a los servicios ambientales (Showalter, 1981; Von Holle \& Motzkin, 2007). Las especies exóticas son las introducidas en un ecosistema fuera de su distribución natural; sin 
IIVESTIGACIÓn Y CIERCIA DE LA UNIVERSIDAD AUTÓNOMA DE AGUASCALIERTES embargo, no todas logran proliferar y su población se restringe. Las especies se tornan invasoras cuando sus características les confieren la capacidad de colonizar, establecerse y causar daños al ambiente (Pimentel, Zuniga, \& Morrison, 2005). Las mismas pueden existir tanto en ecosistemas terrestres como marinos, lo que altera los ciclos biológicos y reduce su productividad (McNeely, 2000).

La introducción de insectos y patógenos representa uno de los mayores problemas por varias razones, entre ellas, su corto ciclo reproductivo y la facilidad de dispersión (Pimentel, Lach, Zuniga, \& Morrison, 2000). Una de las principales vías para la introducción de insectos y patógenos no nativos causantes de plagas y enfermedades es el uso de especies forestales exóticas (Liebhold, Brokerhoff, Garrett, Parke, \& Britton, 2012). En EE. UU., por ejemplo, de 455 especies de insectos y agentes causales de enfermedades forestales no nativas identificadas, 82 (65 especies de insectos y 17 de patógenos) tuvieron como principal vía de traslado la introducción de plantas vivas (Aukema et al., 2010).

Los insectos invasores, además de afectar la biodiversidad, ocasionan graves pérdidas de recursos agrícolas y forestales. En todas partes del mundo la introducción de especies, accidental o intencionalmente (la mayoría con fines de ornato y de control biológico para regular las poblaciones de otros organismos) ha generado resultados negativos (Aukema et al., 2010; Haack, Hérard, Sun, \& Turgeon, 2010). Un ejemplo de esto es la introducción accidental del escarabajo descortezador Dendroctonus valens LeConte en China hacia la década de 1980. Esta especie en EE. UU. no constituye un factor de mortalidad en las diferentes especies de pinos donde se ha reportado (Sun, Miao, Zhang [Zhen], Zhang [Zhongning], \& Gillette, 2004); no obstante, en el país asiático se considera una plaga altamente destructiva en poblaciones de Pinus tabuliformis Carr. -la especie más frecuentemente usada para plantaciones forestales-, ocasionando pérdidas económicas de varios millones de dólares (Yan, Sun, Don, \& Zhang, 2005).

En EE. UU. los insectos y enfermedades exóticas han ocasionado serios problemas económicos y ecológicos. El defoliador Profenusa thompsonii (Konow), introducido de Europa a EE. UU. en la década de 1900, es responsable de la defoliación y mortalidad del arbolado en casos severos de infestación, en extensas áreas urbanas de Alaska
(United Department of Agriculture Forest ServiceAlaska Region, 2010). La escama algodonosa Adelges tsugae (Annand) es un homóptero nativo del este de Asia, cuyos hospederos son diferentes especies de Tsuga (Tsugae spp.) y Picea (Picea spp.). En el año 2007, una evaluación reflejó que el insecto está afectando a la población de Tsugae en cerca de $50 \%$ de su distribución territorial (Rentch, Fajvan, Evans, \& Onken, 2008).

En México, entre las especies mejor documentadas se encuentra la palomilla del nopal (Cactoblastis cactorum). Este insecto fue usado como control biológico en Opuntia spp., en Australia y Sudáfrica, donde algunas especies de este género se consideraban invasoras; sin embargo, posiblemente los fenómenos climáticos ocasionaron su dispersión hacia el continente americano (Zimmermann, Pérez Sandy y Cyen, Gulovob, Soberón, \& Sarukhán, 2000).

La Sierra Fría en Aguascalientes, México, es un macizo montañoso localizado al noroeste del estado, en 1994 esta zona fue decretada como Área Natural Protegida (Gobierno del estado de Aguascalientes, 1994), comprende una superficie de 107,000 ha y corresponde aproximadamente a $20 \%$ del área estatal. A pesar de su estatus, han ocurrido diversas introducciones de organismos, que van desde especies forestales provenientes de otros estados hasta grandes mamíferos originarios de otros países. Aunque no existe un censo de las especies exóticas presentes en la Sierra Fría, algunas observaciones sugieren que en el caso de la vegetación, al menos dos especies de coníferas (Pinus greggii var. greggii Engelm. y Pinus ayacahuite Ehr. var. brachyptera Shaw (Sin; Pinus strobiformis) han sido usadas como una alternativa de reforestación, la primera, por su rápido crecimiento y facilidad de adaptación (MUñoz Flores, Coria Ávalos, García Sánchez, Velasco Bautista, \& Martínez Molina, 2012; Musálem \& Martínez Cantera, 2003), y la segunda, por iniciativa de algunos propietarios. Durante 2016 se encontraron evidencias de una población aislada de un pino identificado posteriormente como $P$. ayacahuite (datos no publicados) en la localidad conocida como "Monte grande" (F. J. Díaz Martínez, comunicación personal); no obstante, se tienen registros de al menos tres predios (rancho La Ciénega, rancho Peña Parada y barranca de Palo Gordo) donde se usó la especie mencionada para reforestación antes de ser identificada, por lo que se le consideró como introducida. 
En 2006 se encontró a Neodiprion omosus Smith, una especie de mosca sierra, infestando sitios reforestados con diferentes especies de pinos, la más afectada (80\%) fue Pinus devoniana Lindl. Fue el primer reporte de este insecto para la Sierra Fría. Solamente se encontraron cuatro puntos de infestación, distribuidos en el predio "El Búfalo" y en la presa "La Araña". En revisiones posteriores no se han reportado daños en más localidades. Asimismo, algunas especies de fauna exótica se han introducido con fines cinegéticos (ie. Elk (Cervus elaphus L.); venado cola blanca texano (Odocoileus virginianus texanus Zimm.), ñu (Connochaetes gnou Lich.), entre otros) (Sosa-Ramírez, Solís-Cámara, Jiménez-Sierra, Íniguez-Dávalos, \& Ortega-Rubio, 2014) y aunque se desconocen con certeza los efectos que pudieran causar en la flora y fauna nativas, algunos autores (Sosa-Ramírez et al., 2014) sugieren que el paisaje puede modificarse y esto pondría en riesgo la permanencia de la diversidad biológica en el área.

P. greggii es una de las especies de coníferas que más se han usado en reforestaciones, tanto en áreas naturales como urbanas. Por ejemplo, en 2011 existían al menos dos proyectos de reforestación de 100 ha cada uno en el uso común del ejido Rincón de Romos. Por otra parte, la introducción de especies exóticas está prohibida en áreas naturales protegidas, como en el caso de la Sierra Fría, por los riesgos que implica para la diversidad nativa. Por la importancia y condición de la especie, es necesario establecer un sistema que permita detectar las posibles plagas y enfermedades que afectan a esta y otras especies y no llegue a convertirse en un riesgo potencial para la diversidad nativa.

\section{MATERIALES Y MÉTODOS}

Detección e identificación del agente causal de daño en una reforestación de $P$. greggii var. greggii Como parte del proyecto "Diagnóstico fitosanitario en comunidades forestales del estado de Aguascalientes" se desarrollaron recorridos en los diferentes ecosistemas del estado. En abril de 2013 se realizaron visitas de diagnóstico en la Sierra Fría en comunidades naturales dominadas por poblaciones de Pinus teocote Schltdl. \& Cham., P. leiophylla Schltdl. \& Cham., P. durangensis Ehren., Quercus potosina Trel., Q. eduardii Trel., Q. sideroxyla Humb. \& Bompl. y asociaciones de ellas. En una visita realizada al predio forestal "Peña Parada", ubicado en las coordenadas UTM 747392 este y 2451219 norte, con una altitud promedio de 2535 m.s.n.m., se revisó un área reforestada con plantas de $P$. greggii var. greggii de dos años de edad (D. Domínguez, comunicación personal), se observaron signos de defoliación y mortalidad de la parte aérea del arbolado. Una revisión de cada espécimen mostró exudaciones sobre el fuste, posiblemente producto de la aparición de insectos con aparato bucal chupador. En el mismo predio se revisaron cinco sitios reforestados con la misma especie de conífera. En el primero, $80 \%$ de los árboles mostraron signos y síntomas de daño por insectos y en $60 \%$ de los casos se encontraron insectos en fase de ninfa, adultos e inmaduros (figura 1). En el segundo sitio se encontró daño por infestación en $30 \%$ de los árboles, en el tercer sitio $40 \%$, en el cuarto sitio $20 \%$ y $30 \%$ en el quinto sitio.

Con la finalidad de encontrar una posible distribución del organismo causante del daño en

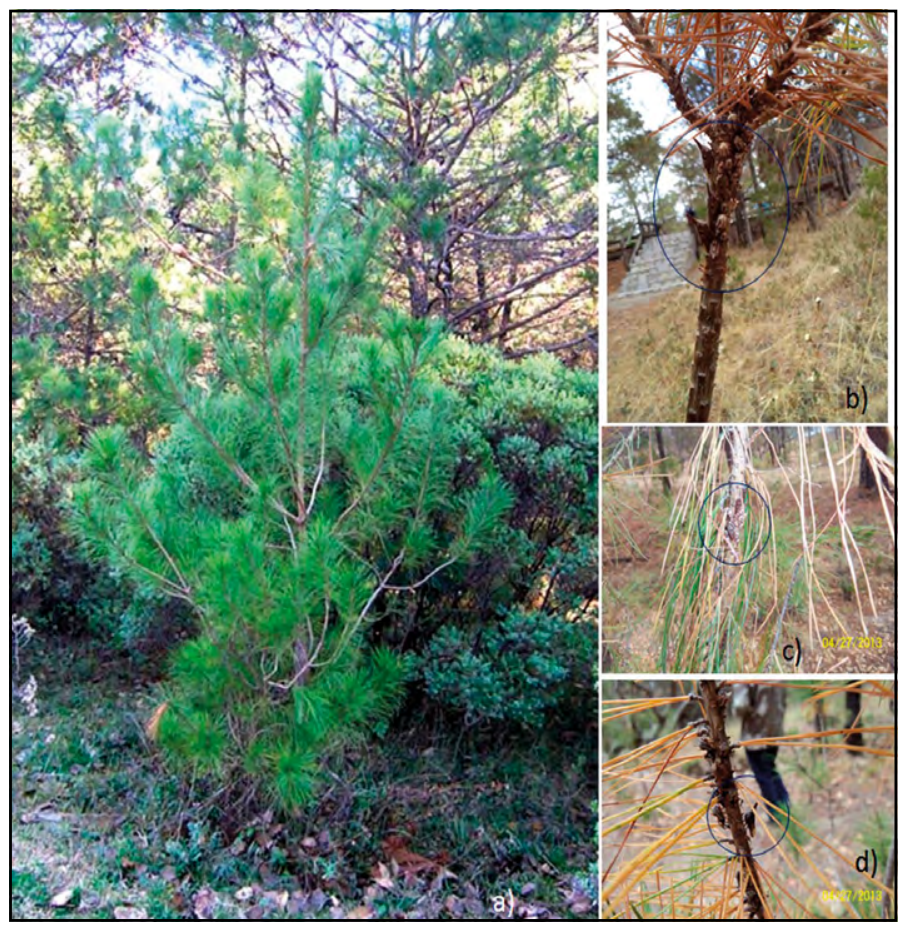

Figura 1. Esquema de daño por A. compressa Walker en plantaciones de $P$. greggii var. greggii en algunos sitios de la Sierra Fría. (a) espécimen sano de $P$. greggii, nótese el follaje en color verde brillante; (b) colonia de individuos adultos de A. compressa (ver círculo en color azul y el follaje amarillento, producido posiblemente por la extracción de savia) sobre el tallo de P. greggii; (c) una colonia de ninfas de A. compressa sobre el tallo de P. greggii, no se aprecian adultos; (d) ejemplares de A. compressa sobre el tallo de P. greggii, se observan algunas ninfas del mismo género.

Imagen cortesía de Vicente Díaz-Núñez. 


\section{INVESTIGAGIÓก Y CIECEIA DE LA UNIVERSIDAD AUTÓNOMA DE AGUASCALIERTES}

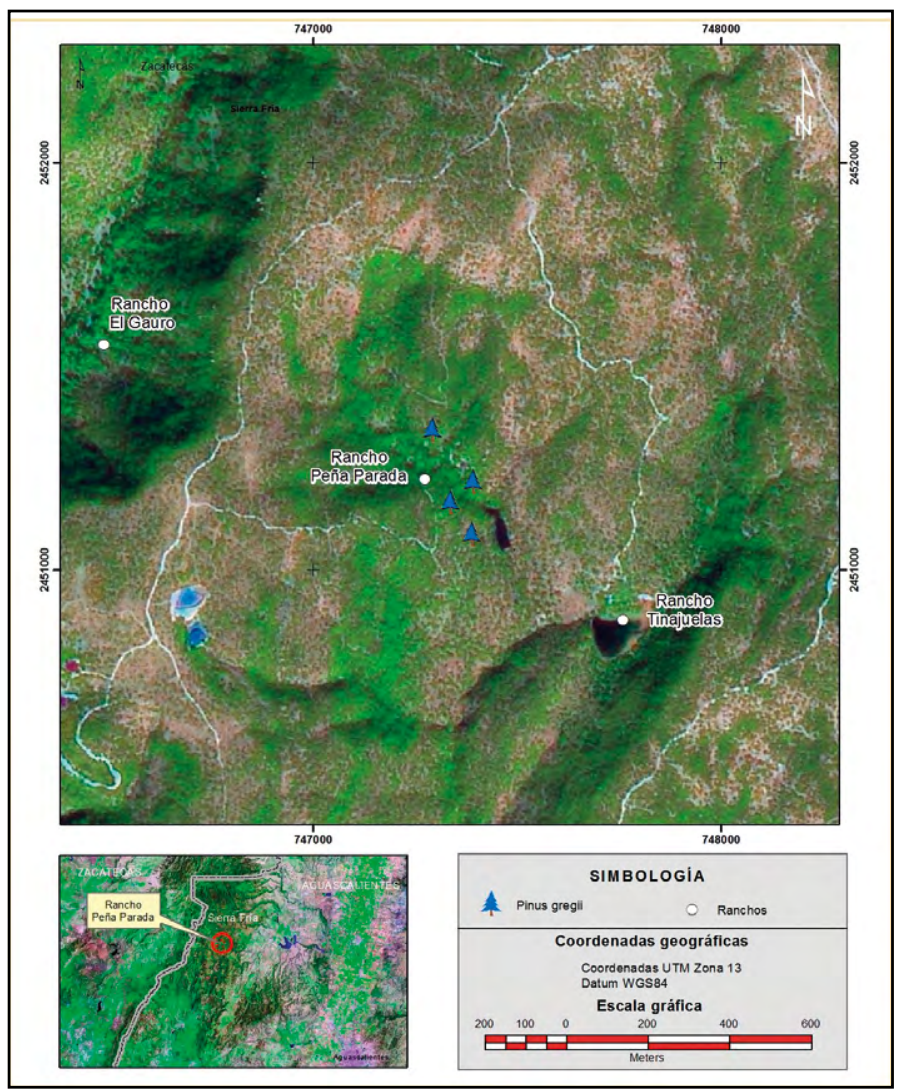

Figura 2. Sitios de reforestación donde se detectó la presencia de A. compressa, afectando poblaciones de P. greggii en la Sierra Fría, Aguascalientes, México.

Imagen cortesía de Vicente Díaz-Núñez.

P. greggii se realizaron recorridos de observación y análisis de los sitios con reforestación en cinco predios de la Sierra Fría: (a) Barranca Piletas, (b) Barranca de Juan Francisco, (c) Predio el Búfalo, y Rancho San Francisco (figura 2).

En ninguno de los sitios de trabajo se identificaron signos y síntomas de daños por el organismo detectado en el primer sitio. Para la identificación se realizaron colectas de ninfas y adultos, mismas que fueron colocadas en frascos con alcohol a $70 \%$ para su almacenamiento y posterior identificación. La identificación se llevó a cabo con base en literatura especializada (Day, 1999; Wallace \& Deitz, 2004); sin embargo, se contó con la colaboración de Stuart McKamey, adscrito al Servicio de Investigación Agrícola del Departamento de Agricultura de los Estados Unidos (McKamey, 2007).

\section{Monitoreo de reforestaciones con $P$. greggii}

Durante los años 2014, 2015, y 2016 se realizaron dos visitas anuales, al inicio de la temporada de lluvias, en el mes de junio, y durante la temporada de temperaturas más altas, correspondiente al mes de mayo (CONAGUA, 2017) en el predio donde originalmente se localizó el insecto, así como en el área de bienes comunales del ejido Rincón de Romos, donde en 2011 se realizó una reforestación con $P$. gregii en una superficie de 100 ha. En esta última se establecieron 10 sitios de muestreo de $500 \mathrm{~m}^{2}$ representativos de la parcela y distribuidos aleatoriamente.

\section{RESULTADOS}

De acuerdo con la estructura de varias muestras de adultos y ninfas tomadas en campo y corroboradas en laboratorio mediante el uso de un estereoscopio, los insectos analizados corresponden al género Aconophora (Hemiptera: Membracidae), específicamente a Aconophora compressa Dietrich, una especie de insecto chupador comúnmente conocido como periquito de la lantana, relacionado como agente de control biológico de Lantana camara L. (lantana) (Verbenacea), una especie arbustiva considerada como nociva para las actividades ganaderas por su capacidad invasora en condiciones naturales. Se muestran figuras ilustrativas de A. compressa (figura 3).

En el monitoreo realizado no se encontraron evidencias de infestación; sin embargo, en 2015 se encontró un solo ejemplar adulto de A. compressa en la localidad ejidal de Rincón de Romos. En el muestreo de 2016 no existió infestación en ninguna de las localidades analizadas.

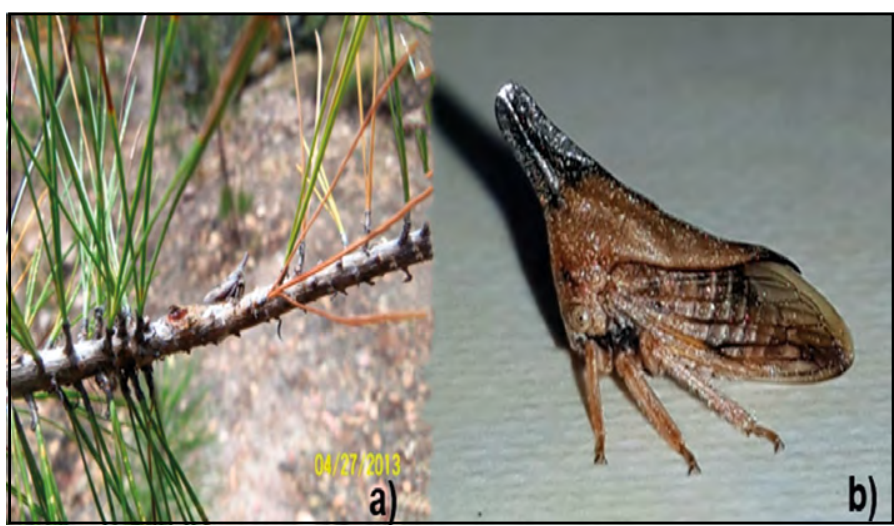

Figura 3. Ejemplares de A. compressa. a) Adulto de A. compressa posado sobre una ramilla de $P$. greggii en la Sierra Fría. b) Acercamiento de un ejemplar adulto de A. compressa. Imágenes cortesía de Vicente Díaz-Núñez. 


\section{IIVESTIGAGIÓn Y CUERCIA DE LA UกIVERSIDAD AUTÓnOMA DE RGUASCALIERTES}

\section{DISCUSIÓN}

En muchos de los estudios de caso que se han analizado, tanto en México como en el extranjero, la introducción de especies exóticas con diferentes fines (ornato, restauración, control biológico) han sido más perjudiciales que benéficas (Pimentel et al., 2005) y se ha recurrido a prácticas de manejo alternativas para reducir el daño que ocasionan a la diversidad nativa (Dahlsten et al., 2005; Haack et al., 2010).

En el caso de las áreas naturales protegidas, la normatividad existente indica que no debe permitirse el ingreso de especies ajenas al sitio, pues existe el riesgo de que se introduzcan y proliferen organismos asociados (Ortiz-Monasterio, 2014), que bajo las condiciones propias de la especie introducida sus efectos sean mínimos, pero que en otras condiciones ambientales su población se torne dañina (Simberloff \& Von Holle, 1999). Por otra parte, debe evitarse el uso de especies exóticas de flora y fauna y darse prioridad a las especies nativas, tanto en las iniciativas de los propietarios (en este caso, reforestaciones y plantaciones con fines comerciales) como en los programas gubernamentales establecidos por los diferentes niveles de gobierno.

Existen algunos casos documentados sobre el daño causado por los insectos del género Aconophora en comunidades y poblaciones para el control de plantas arvenses (Palmer, Wilson, \& Pullen, 1996; The State of Queensland DEEDI, 2010). En EE. UU., desde 1995 se ha estado evaluando el uso de A. compressa para el control de varias especies de plantas exóticas e invasoras; sin embargo, aún no se ha analizado si esto pudiera traer efectos adversos (Manners, Walter, Palmer, Dhileepan, \& Hastwell, 2006). En México, la misma especie fue introducida en el estado de Morelos como un método de control biológico de las poblaciones de L. camara L. (Verbenaceae), una especie de planta silvestre considerada como invasora para los pastizales (Palmer et al., 1996); sin embargo, se han encontrado registros de especímenes ovipositando sobre Juniperus spp. y Jacaranda spp., aunque no representan daños potenciales para estas especies (Palmer et al., 1996).

La especie de Aconophora detectada en la Sierra Fría, Aguascalientes, representa el primer caso reportado en una especie forestal, tampoco se

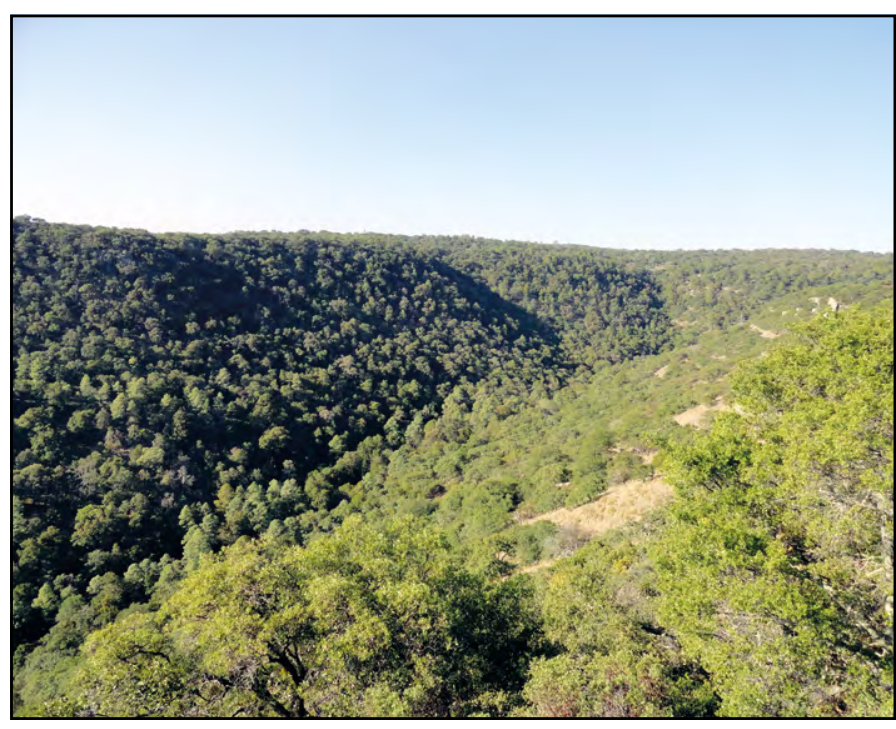

Figura 4. Cañada de piletas, Sierra Fría, Aguascalientes. Paisaje dominado por $P$. leiophylla, P. teocote y $Q$. sideroxylla. Imagen cortesía de Vicente Díaz-Núñez y Joaquín Sosa-Ramírez.

tienen reportes de su presencia en plantas arvenses de bosque templado y bosque tropical seco en la entidad. En el caso de los reportes de la aparición de mosca sierra (2006) y de A. compressa (2013), es probable que en los hospederos $P$. devoniana y $P$. greggii var. greggii, respectivamente, la infestación se haya debido a la introducción de especímenes infestados donados por la Comisión Nacional Forestal local, pero producidos en otro estado de la república, por lo que la primera acción que debe realizarse es una rigurosa inspección para verificar que las plantas usadas con fines de reforestación y restauración estén sanas.

A pesar del estatus de área natural protegida que presenta la Sierra Fría (figura 4), por su rápido crecimiento, en años pasados se usó la especie $P$. greggii en algunas localidades para reforestar áreas afectadas principalmente por el daño de escarabajos descortezadores del género Dendroctonus, como una iniciativa de los propietarios del área, no como parte de los programas auspiciados por los gobiernos federal y estatal (Secretaría de Medio Ambiente y Recursos Naturales, Comisión Nacional Forestal, Comisión Nacional de Áreas Naturales Protegidas, Secretaría de Medio Ambiente del Estado, entre otras), aunque en los recorridos realizados no se ha encontrado regenerado natural de esta especie, probablemente esto indique que no se ha reproducido y que, muy probablemente, desaparecerá del ecosistema de manera natural. En los predios recorridos, solamente se ha encontrado al 
IIVESTIGACIÓn Y CIERCIA DE LA UNIVERSIDAD AUTÓNOMA DE RGUASCALIERTES membrácido afectando poblaciones de P. greggii; sin embargo, no se descarta la posibilidad de que el insecto pueda dispersarse hacia otras comunidades de coníferas, específicamente en regenerados, reforestaciones y plantaciones comerciales, por lo que es importante que investigadores, especialistas en sanidad forestal y sobre todo los propietarios y responsables del manejo de cada uno de los predios realicen monitoreo para detectar áreas con posible daño.

\section{CONCLUSIONES}

El insecto chupador identificado corresponde a $A$. compressa, su hospedero principal son las plantas arvenses y es el primer registro en la zona afectando a una especie forestal. En la Sierra Fría, su hospedero es $P$. gregii var. greggii, una especie de conífera exótica que se ha usado en programas de reforestación, aunque no tiene una distribución amplia en el paisaje forestal. Es importante continuar con el monitoreo para detectar probables infestaciones y su dispersión hacia otras comunidades de pinos.

\section{Agradecimientos}

Los autores reconocen el apoyo brindado por la Secretaría de Medio Ambiente del Estado de Aguascalientes, así como por la Comisión Nacional Forestal. Se agradece a Francisco Javier Díaz Martínez, Luis Alberto Hernández Gaytán y Marcos Ruiz Esteban por su participación en el trabajo de campo. Se agradece el financiamiento otorgado por la Comisión de Medio Ambiente y Recursos Naturales del Congreso de la Unión (COMARNAT) a través del proyecto original "Manejo Fitosanitario y Recuperación de Zonas en Ecosistemas Perturbados, en la Sierra Fría, Aguascalientes, México".

\section{REFERENCIAS}

- Aukema, J. E., McCullough, D. G., Von Holle, B., Liebhold, A. M., Britton, K., \& Frankel, S. J. (2010). Historical accumulation of nonindigenous forest pests in the Continental United States. Bioscience, 60(1 1), 886-897. doi: 10.1525/bio.2010.60.11.5

- Comisión Nacional del Agua. (2017). Información climatológica del estado de Aguascalientes. 4 de Julio de 2017 [Portal electrónico]. Recuperado de http://smn.cna. gob.mx/tools/RESOURCES/Normales51 10/NORMAL01 103.TXT.

- Dahlsten, D. L., Daane, K. M., Paine, T. D., Sime, K. R., Lawson, A. B., Rowney, D. L., ..., Taylor, G. (2005). Imported parasitic wasp helps control red gum lerp psyllid. California Agriculture, 59(4), 229-234.

- Day, M. F. (1999). The genera of Australian Membracidae (Hemiptera: Auchenorrhyncha). Invertebrate Taxonomy 13(4), 629-747.

- Gobierno del estado de Aguascalientes. (1994). Periódico oficial del estado de Aguascalientes, Tomo LVII, no. 5.

- Haack, R. A., Hérard, F., Sun, J., \& Turgeon, J. J. (2010). Managing invasive populations of Asian longhorned beetle and citrus longhorned beetle: A worldwide perspective. Annual Review of Entomology, 55, 521-546.

- Liebhold, A. M., Brokerhoff, E. G., Garrett, L. J., Parke, J. L., \& Britton, K. O. (2012). Live plant imports: The major pathway for forest insect and pathogen invasions of the US. Frontiers in Ecology and the Environment 10(3), 135-143.
- Manners, A. G., Walter, G. H., Palmer, W. A., Dhileepan, K., \& Hastwell, G. T. (2006). Retrospective host testing of Aconophora compressa: Reproduction and survival on the target weed, Lantana camara, and non-target species. En C. Preston, J. H. Watts, \& N. D. Crossman (Eds.), Managing weeds in a changing climate: Proceedings of the Fifteenth Australian Weed Conference (pp. 595-598). South Australia: Weed Management Society of South Australia.

- McKamey, S. H. (2007). Taxonomic catalogue of the leafhoppers (membracoidea). Part 1. Cicadellinae. Memoirs of the American Entomological Society, 78, 1-394.

- McNeely, J. A. (2000). The future of alien-invasive species: Changing social views. En H. A. Mooney \& R. J. Hoobs (Eds.), Invasive species in a changing world (350 pp.). Washington, DC: Island Press.

- Muñoz Flores, H. J., Coria Ávalos, V. M., García Sánchez, J. J., Velasco Bautista, E., \& Martínez Molina, G. (2012). Evaluación de una plantación de Pinus greggii (Emgelm.) con dos espaciamientos. Revista Mexicana de Ciencias Forestales, $3(11), 57-70$.

- Musálem, M. A., \& Martínez Cantera, G. (2003). Monografía de Pinus greggii Engelm. Libro técnico no. 9 (341 pp.). El Horno, Chapingo, Estado de México, México: INIFAP-SAGARPACONACYT-CONABIO.

- Ortiz-Monasterio, A. (2014). Gestión de las especies exóticas invasoras: Análisis de la legislación mexicana. En R. Mendoza y 


\section{InVESTIGACIÓn Y CIERCIR DE LA UחIVERSIDAD AUTÓnOMH DE RGUASCHLIETTES}

P. Koleff (Coords.), Especies acuáticas invasoras en México (pp. 169-184). México: Comisión Nacional para el Conocimiento y Uso de la Biodiversidad.

- Palmer, W. A., Wilson, B. W., \& Pullen, K. R. (1996). The hostrange of Aconophora compressa Walker (Homoptera: Membracidae): A potential biological control agent for Lantana camara L. (Verbenaceae). Proceedings of the Entomological Society of Washington, 98, 617-624.

- Pimentel, D., Lach, L., Zuniga, R., \& Morrison, D. (2000). Environmental and economic costs of nonindigenous species in the United States. BioSciences, 50(1), 53-65.

- Pimentel, D., Zuniga, R., \& Morrison, D. (2005). Update on the environmental and economic costs associated with alieninvasive species in the United States. Ecological Economics, 52(3), 273-288.

- Rentch, J., Fajvan, M. A., Evans, R. A., \& Onken, B. (2008). Using dendrochronology to model hemlock woolly adelgid effects on eastern hemlock growth and vulnerability. Biological Invasions 11, 551-563. doi: 10.1007/s10530-008-9270-x

- Showalter, T. D. (1981). Insect herbivore relationship to the state of the host plant: Biotic regulation of ecosystem nutrient cycling through ecological succession. Oikos, 37(1), 126-130.

- Simberloff, D., \& Von Holle, B. (1999). Positive interactions of nonindigenous species: Invasional meltdown. Biological invasions 1 (1), 21-32.

- Sosa-Ramírez, J., Solís-Cámara, A. B., Jiménez-Sierra, C. L., Íñiguez-Dávalos, L. I., \& Ortega-Rubio, A. (2014). Manejo del Área Natural Protegida Sierra Fría, Aguascalientes: Situación actual y desafíos. Investigación y Ciencia de la Universidad Autónoma de Aguascalientes, 22 (60), 71-77.
- Sun, J., Miao, Z., Zhang, Z. [Zhen], Zhang, Z. [Zhongning], \& Gillette, N. E. (2004). Red turpentine beetle, Dendroctonus valens Le Conte (Coleoptera: Scolytidae) response to host semiochemicals in China. Environmental Entomology, 33, 206212.

- The State of Queensland, Department of Employment, Economic Development and Innovation. (2010). Aconophora on fiddlewood trees. Aconophora compressa [Hoja informativa]. Recuperada de http://www.arboroperations. com.au/wp-content/uploads/2014/08/aconophora-onfiddlewood-trees-deedi-factsheet.pdf

- United States Department of Agriculture-Forest Service (Alaska Region). (2010). Invasive exotic insect species: Amber marked birch leaf miner [Reporte breve]. Recuperado de http://www. fs.usda.gov/Internet/FSE_DOCUMENTS/stelprdb5299799.pdf

- Von Holle B., \& Motzkin, G. (2007). Historical land use and environmental determinants of nonnative plant distribution in coastal southern New England. Biological Conservation, 136(1), 33-43.

- Wallace, M. S., \& Deitz, L. L. (2004). Phylogeny and systematics of the treehopper subfamily Centrotinae (Hemiptera: Membracidae). En Memoirs on Entomology, International (Vol. 19, 377 pp.). FL: Associated Publishers.

- Yan, Z., Sun, J., Don, O., \& Zhang, Z. (2005). The red turpentine beetle, Dendroctonus valens Le Conte (Scolytidae): An exotic invasive pest of pine in China. Biodiversity \& Conservation, $14(7), 1735-1760$.

- Zimmermann, H. G., Pérez Sandy y Cyen, M., Gulovob, J., Soberón, J., \& Sarukhán, J. (2000). Cactoblastis cactorum, una nueva plaga de muy alto riesgo para las opuntias de México. CONABIO Biodiversitas, 6(33), 1-14. 